УДК 547.313: 544.188

DOI: $10.35211 / 1990-5297-2020-12-247-35-38$

\author{
В. А. Бабкин ${ }^{1}$, Д. С. Андреев ${ }^{1}$, А. В. Игнатов ${ }^{1}$, Д. А. Борисов ${ }^{1}$ Р. О. Болдырев ${ }^{1}$, Т. В. Крапчетова ${ }^{I}$, \\ М. В. Решетникова ${ }^{1}$, М. Н. Киселева ${ }^{1}$, Е. С. Титова ${ }^{2,3}$, А. И. Рахимов ${ }^{2}$ В. С. Белоусова ${ }^{4}$
}

РАСЧЕТ ЭЛЕКТРОННОЙ СТРУКТУРЫ МОНОМЕРОВ КАТИОННОЙ ПОЛИМЕРИЗАЦИИ, РАЗВЕТЛЕННЫХ В В-ПОЛОЖЕНИИ ПО ОТНОШЕНИЮ К ДВОЙНОЙ СВЯЗИ, МЕТОДОМ ФFТ

\footnotetext{
${ }^{1}$ Себряковский филиал Волгоградского государственного технического университета

${ }^{2}$ Волгоградский государственный технический университет

${ }^{3}$ Волгоградский государственный медицинский университет Минздрава России

${ }^{4}$ Первый Московский государственный медицинский университет

им. И. М. Сеченова Минздрава России (Сеченовский Университет)
}

E-mail: titova051@rambler.ru

В настоящей работе выполнен квантово-химический расчет некоторых мономеров: 4-метилпентен-1, 4метилгексен-1, 4,4-диметилпентен-1 методом функционала плотности DFT. Получено оптимизированное геометрическое и электронное строение этих соединений. Установлено, что изучаемые мономеры относятся к классу очень слабых СН-кислот (рКа $=28)$.

Ключевые слова: квантово-химический расчет, метод DFT, 4-метилпентен-1, 4-метилгексен-1, 4,4-диметилпентен-1, кислотная сила.

\section{Введение}

Классическими мономерами катионной полимеризации являются мономеры, разветвленные в $\beta$-положении: 4-метилпентен-1, 4-метилгексен-1, 4,4-диметилпентен-1.

Детали полимеризации 4-метилпентена-1 в присутствии кислот Льюиса, в частности, $\mathrm{AlCl}_{3}$ в растворе метилхлорида изучал Дж. Кеннеди [1]. Интересно, что полимер максимального молекулярного веса был получен при $-78{ }^{\circ} \mathrm{C}$ $\left(\mathrm{M}_{\mathrm{v}}=2 \cdot 10^{6}\right)$, а при температурах более низких образуются продукты с меньшим молекулярным весом. Структуру поли-4-метилпентена-1, полученного катионным способом, изучали методом ЯМР-спектроскопии в различных работах [2-5].

Полимер 4-метилгексена-1 самого высокого молекулярного веса был получен при температуре $-78{ }^{\circ} \mathrm{C}\left(\mathrm{M}_{\mathrm{v}}=273000\right)$ и является аморфным каучуком с $\mathrm{T}_{\text {ст }}=-23^{\circ} \mathrm{C}[6]$. Структуру этого каучукоподобного полимера исследовали методом ЯМР- и ИК-спектроскопии. [1]

Впервые полимеризация 4,4-диметилпентена-1 описана в работе [2] в 1963 году, в которой было отмечено, что после полимеризации этого олефина в присутствии твердого $\mathrm{AlCl}_{3}$ при $0{ }^{\circ} \mathrm{C}$.

В работе [7] в качестве катализатора использовали $\mathrm{AlCl}_{3}$ в растворителе этилхлориде и вели процесс при температуре -78 и $130{ }^{\circ} \mathrm{C}$. Полученный продукт - твердое клейкое веще- ство с молекулярной массой $\mathrm{M}_{\mathrm{n}}=1400$. По данным ЯМР- и ИК-спектроскопии, этот полимер состоит из звеньев, которые должны образовываться в результате следующих одна за другой миграций гидридных и метильных ионов [1].

Несмотря на то что эти соединения были синтезированы еще XIX-XX веках [8], их квантово-химический расчет методом DFT не выполнялся. В связи с этим, цель данной работы квантово-химический расчет вышеперечисленных мономеров методом DFTPBE0 в базисе 3$21 \mathrm{G}$ по методике и с использованием программ [9-11], аналогично [12] с оптимизации геометрии по всем параметрам стандартным градиентным методом. Общий заряд изучаемых молекулярных систем в основном состоянии был равен 0 , а мультиплетность 1, так как в основном состоянии молекул все электроны спарены, и суммарный спин равен 0 . Поэтому $\mathrm{M}=2 \mathrm{~S}+1=1$.

\section{Результаты расчетов}

Для 4-метилпентена-1 оптимизированные длины связей между атомами углерода в кольце находятся в диапазоне 1.33 - $1.55 \AA$, С-Н 1.09 $1.10 \AA$. Оптимизированные значения валентных углов (в градусах) получены соответственно следующие: С(1)-С(2)-С(3)-124.91, С(2)-С(3)$\mathrm{C}(4)-112.19, \mathrm{C}(3)-\mathrm{C}(4)-\mathrm{C}(5)-110.23, \mathrm{C}(3)-\mathrm{C}(4)-$ $\mathrm{C}(6)-110.03, \mathrm{C}(5)-\mathrm{C}(4)-\mathrm{C}(6)-110.46, \mathrm{H}(8), \mathrm{C}(1)-$ $\mathrm{C}(2)-121.93, \mathrm{H}(7)-\mathrm{C}(1)-\mathrm{C}(2)-121.37, \mathrm{C}(1)-\mathrm{C}(2)-$ $\mathrm{H}(9), 119.67, \mathrm{C}(3)-\mathrm{C}(2)-\mathrm{H}(9)-115.41, \mathrm{C}(2)-\mathrm{C}(3)-$ H(10)-109.42, C(2)-C(3)-H(11)-110.14, C(3)-C(4)-

(С Бабкин В. А., Андреев Д. С., Игнатов А. В., Борисов Д. А., Болдырев Р. О., Крапчетова Т. В., Решетникова М. В., Киселева М. Н., Титова Е. С., Рахимов А. И., Белоусова В. С., 2020. 
$\mathrm{H}(12)-108.14, \quad \mathrm{C}(4)-\mathrm{C}(6)-\mathrm{H}(12)-\quad 108.92, \quad \mathrm{C}(4)-$ C(5)-H(12)-108.96, C(4)-C(3)-H(11)-108.72, C(4)C(3)-H(12)-108.52, C(4)-C(5)-H(13)-110.86, C(4)C(5)-H(14)-110.76, C(4)-C(5)-H(15)-109.61, C(4)$\mathrm{C}(6)-\mathrm{H}(16)-109.83, \mathrm{C}(4)-\mathrm{C}(6)-\mathrm{H}(17)-111.08, \mathrm{C}(4)-$ $\mathrm{C}(6)-\mathrm{H}(18)-110.80$.

Заряды на атомах: C(1)-(-0.429), C(2)-(-0.183), C(3)-(-0.453), C(4)-(-0.300), C(5)-(-0.595), C(6)-($0.596), \mathrm{H}(7)-(0.206), \mathrm{H}(8)-(0.209), \mathrm{H}(9)-(0.212)$, H(10)-(0.226), H(11)-(0.216), H(12)-(0.219), H(13)(0.208), H(14)-(0.226), H(15)-(0.204), H(16)(0.209), Н(17)-(0.209), Н(18)-(0.211) (рис. 1).

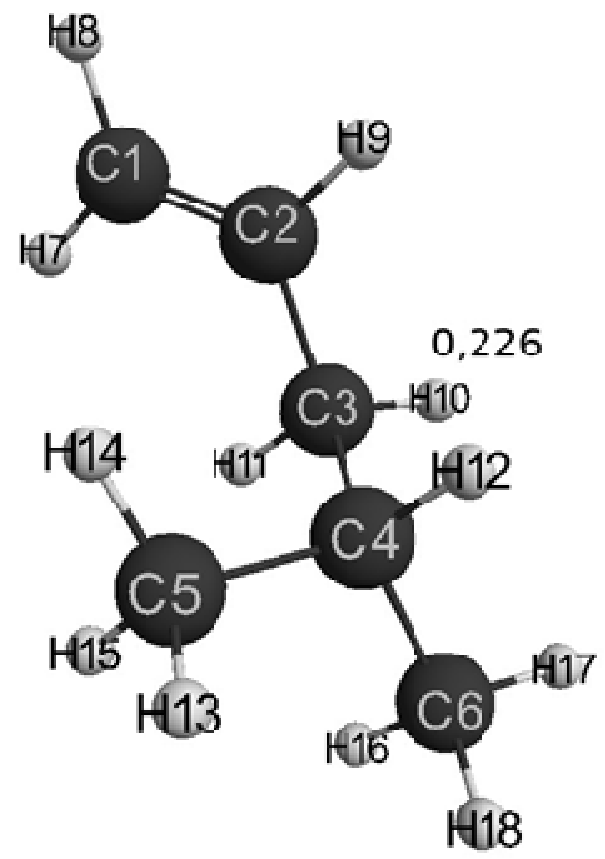

Рис. 1. Геометрическое и электронное строение молекулы 4-метилпентена-1

Для 4-метилгексена-1 оптимизированные длины связей между атомами углерода в кольце находятся в диапазоне 1,33-1.55 $\AA$, С-Н 1.09 $1.10 \AA$ А̊. Оптимизированные значения валентных углов (в градусах) получены соответственно следующие: C(1)-C(2)-C(3)-124.44, C(2)-C(3)$\mathrm{C}(4)-112.90, \quad \mathrm{C}(3)-\mathrm{C}(4)-\mathrm{C}(5)-110.04, \quad \mathrm{C}(3)-\mathrm{C}(4)-$ $\mathrm{C}(6)-111.22, \quad \mathrm{C}(5)-\mathrm{C}(4)-\mathrm{C}(6)-111.63, \quad \mathrm{C}(4)-\mathrm{C}(6)-$ $\mathrm{C}(7)-112.81, \mathrm{H}(8)-\mathrm{C}(1)-\mathrm{C}(2)-121.36, \mathrm{H}(9)-\mathrm{C}(1)-$ $\mathrm{C}(2)-121.97, \mathrm{C}(1)-\mathrm{C}(2)-\mathrm{H}(10)-119.47, \mathrm{C}(3)-\mathrm{C}(2)-$ $\mathrm{H}(10)-116.08, \mathrm{C}(2)-\mathrm{C}(3)-\mathrm{H}(11)-109.40, \mathrm{C}(2)-\mathrm{C}(3)-$ $\mathrm{H}(12)-109.43, \mathrm{C}(3)-\mathrm{C}(4)-\mathrm{H}(14)-107.18, \mathrm{C}(5)-\mathrm{C}(4)-$ $\mathrm{H}(14)-108.88, \mathrm{C}(6)-\mathrm{C}(4)-\mathrm{H}(14)-107.74, \mathrm{C}(4)-\mathrm{C}(5)-$ $\mathrm{H}(13)-109.73, \mathrm{C}(4)-\mathrm{C}(5)-\mathrm{H}(15)-110.56, \mathrm{C}(4)-\mathrm{C}(5)-$ $\mathrm{H}(16)-111.40, \mathrm{C}(4)-\mathrm{C}(6)-\mathrm{H}(17)-108.84, \mathrm{C}(4)-\mathrm{C}(6)-$ $\mathrm{H}(18)-109.06, \mathrm{C}(7)-\mathrm{C}(6)-\mathrm{H}(17)-109.00, \mathrm{C}(7)-\mathrm{C}(6)-$ $\mathrm{H}(18)-109.60, \mathrm{C}(6)-\mathrm{C}(7)-\mathrm{H}(19)-111.15, \mathrm{C}(6)-\mathrm{C}(7)-$ $\mathrm{H}(20)-110.90, \mathrm{C}(6)-\mathrm{C}(7)-\mathrm{H}(21)-110.34$.
Заряды на атомах: C(1)-(-0.425), C(2)-(0.187), C(3)-(-0.462), C(4)-(-0.272), C(5)-(-0.603), $\mathrm{C}(6)-(-0.421), \mathrm{C}(7)-(-0.629), \mathrm{H}(8)-(0.205), \mathrm{H}(9)-$ (0.209), H(10)-(0.215), H(11)-(0.224), $\mathrm{H}(12)-$ (0.219), H(13)-(0.223), $\quad \mathrm{H}(14)-(0.218), \quad \mathrm{H}(15)-$ (0.207), H(16)-(0.211), $\quad \mathrm{H}(17)-(0.211), \quad \mathrm{H}(18)-$ (0.216), H(19)-(0.216), H(20)-(0.214), $\mathrm{H}(21)-$ (0.211) (рис. 2).

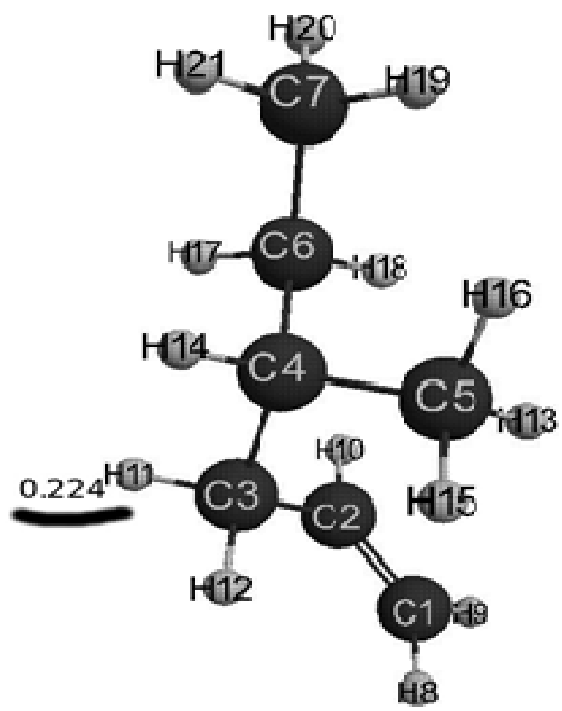

Рис. 2. Геометрическое и электронное строение молекулы 4-метилгексена-1

Для 4,4-диметилпентена-1 оптимизированные длины связей между атомами углерода в кольце находятся в диапазоне 1,33-1.55 $\AA$, CН $1.09-1.10 \AA$ А. Оптимизированные значения валентных углов (в градусах) получены соответственно следующие: C(1)-C(2)-C(3)-124.29, $\mathrm{C}(2)-\mathrm{C}(3)-\mathrm{C}(4)-113.90, \quad \mathrm{C}(3)-\mathrm{C}(4)-\mathrm{C}(5)-109.71$, $\mathrm{C}(3)-\mathrm{C}(4)-\mathrm{C}(6)-110.57, \quad \mathrm{C}(3)-\mathrm{C}(4)-\mathrm{C}(7)-108.18$, $\mathrm{C}(5)-\mathrm{C}(4)-\mathrm{C}(6)-109.82, \quad \mathrm{C}(5)-\mathrm{C}(4)-\mathrm{C}(7)-109.44$, $\mathrm{C}(6)-\mathrm{C}(4)-\mathrm{C}(7)-109.09, \quad \mathrm{C}(2)-\mathrm{C}(1)-\mathrm{H}(8)-121.99$, $\mathrm{C}(2)-\mathrm{C}(1)-\mathrm{H}(9)-121.31, \quad \mathrm{C}(1)-\mathrm{C}(2)-\mathrm{H}(10)-119.48$, C(3)-C(2)-H(10)-116.23, C(2)-C(3)-H(11)-109.53, $\mathrm{C}(2)-\mathrm{C}(3)-\mathrm{H}(12)-109.53, \mathrm{C}(4)-\mathrm{C}(3)-\mathrm{H}(11)-108.23$, C(4)-C(3)-H(12)-107.78, C(4)-C(5)-H(13)-110.22, C(4)-C(5)-H(14)-110.33, C(4)-C(5)-H(21)-110.44, C(4)-C(6)-H(17)-109.93, C(4)-C(6)-H(18)-110.59, $\mathrm{C}(4)-\mathrm{C}(6)-\mathrm{H}(19)-111.01, \mathrm{C}(4)-\mathrm{C}(7)-\mathrm{H}(15)-110.21$, $\mathrm{C}(4)-\mathrm{C}(7)-\mathrm{H}(16)-110.60, \mathrm{C}(4)-\mathrm{C}(7)-\mathrm{H}(20)-110.51$.

Заряды на атомах: C(1)-(-0.425), C(2)-($0.190), C(3)-(-0.423), C(4)-(-0.207), C(5)-(-0.564)$, $\mathrm{C}(6)-(-0.572), \mathrm{C}(7)-(-0.562), \mathrm{H}(8)-(0.209), \mathrm{H}(9)-$ (0.205), H(10)-(0.215), H(11)-(0.216), H(12)(0.221), H(13)-(0.204), H(14)-(0.203), H(15)(0.208), H(16)-(0.206), H(17)-(0.210), H(18)(0.205), H(19)-(0.213), H(20)-(0.207), $\mathbf{H ( 2 1 ) -}$ (0.223) (рис. 3). 


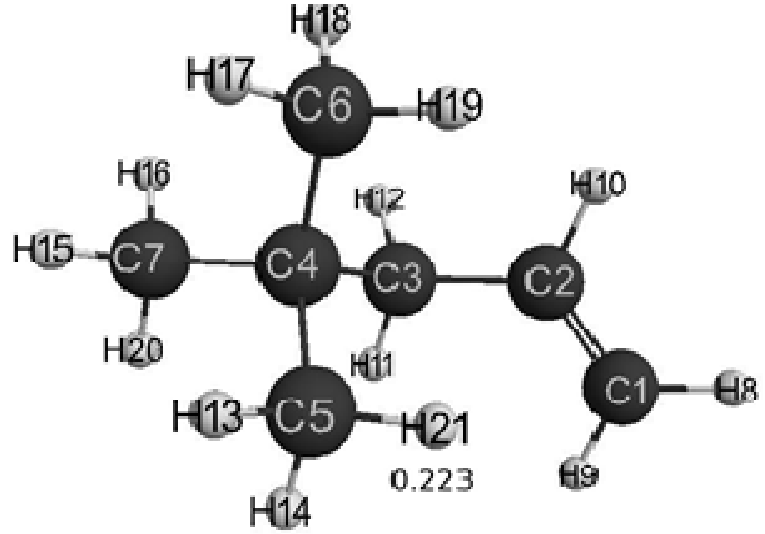

Рис. 3. Геометрическое и электронное строение молекулы 4,4-диметилпентена-1

Используя формулу [13] для метода DFT: $\mathrm{PBE0} / 3-21 \mathrm{G}-\mathrm{pKa}=51.52-103.867 q_{\max } \mathrm{H}^{+}$ $\left(q_{\max }{ }^{\mathrm{H}^{+}}=+0.226, \quad\right.$ (табл. 1$), q_{\max }{ }^{\mathrm{H}^{+}}=+0.224$, $q_{\max }{ }^{+}=+0.223$, где $q_{\max }{ }^{\mathrm{H}^{+}}-$максимальный заряд на атоме водорода, $\mathrm{pКа} \mathrm{-} \mathrm{универсальный} \mathrm{пока-}$ затель кислотности, находим значение кислотной силы $\mathrm{pKa}=28$.

Квантово-химические параметры ( $\left.\mathrm{E}_{0}, \mathrm{q}_{\max }{ }^{\mathrm{H}+}\right)$ и (рКа) исследуемых мономеров

\begin{tabular}{|l|c|c|c|}
\hline \multicolumn{1}{|c|}{ Мономер } & $\mathrm{E}_{0, \text { кДж/моль }}$ & $\mathrm{q}_{\max }^{\mathrm{H}^{+}}$ & $\mathrm{pKa}$ \\
\hline 4-метилпентен-1 & -614970 & +0.226 & 28 \\
\hline 4-метилгексен-1 & -717479 & +0.224 & 28 \\
\hline 4,4-диметилпентен-1 & -717492 & +0.223 & 28 \\
\hline
\end{tabular}

Таким образом, получены оптимизированные атомно-молекулярные структуры изучаемых мономеров методом DFT. Рассчитаны квантово-химические параметры $\left(\mathrm{E}_{0}, \mathrm{q}_{\max }{ }^{++}\right)$, распределение электронной плотности на атомах, которая находится в полном соответствии с законом сохранения заряда и др. Доказано, что исследуемые мономеры относятся к классу очень слабых СН-кислот (рКа > 14).

\section{БИБЛИОГРАФИЧЕСКИЙ СПИСОК}

1. Кеннеди, Дж. Катионная полимеризация олефинов / Дж. Кеннеди. - М., 1978. - 431 c.

2. Edwards, W. R. Carbonium ion rearrangement in the cationic polymerization of branched alpha olefins / W. R. Edwards, Chamberlain N.F. // Journal of Polymer Science Part A: General Papers. - 1963. - V1. - Issue 7. - P. 2299-2308.

3. Goodrich, J.E. Cationic polymerization of 4-methyl-1pentene. / J.E. Goodrich, R. S. Porter // Journal of Polymer Science Part B: Polymer Letters. - 1964. - V 2. - I 4. p. 353-357.

4. Wanless, G.G. Intramolecular hydride shift polymerization by cationic mechanism III-Structure analyses of deuterated and non-deuterated poly-4-methyl-1-pentene /
G.G. Wanless, J. P. Kennedy // Polymer. - 1965. - V. 6. p. 111-174.

5. Ketley, A.D. Isomerization polymerization of branched alpha olefins. / A.D. Ketley // Journal of Polymer Science Part B: Polymer Letters. - 1964. - V 2. - I 8. - p. 827-831.

6. Kennedy, J.P., Elliot J.J., Naegele W. Intramolecular hydride shift polymerization by cationic mechanism VII. The polymerization of 4 methyl 1 hexene / J.P Kennedy, J.J. Elliot, W. Naegele. // Journal of Polymer Science Part B: Polymer Letters. - 1965. - V 3. - I 9. - p. 729-734.

7. Sartori, G. A polymerization involving hydrogen and methyl group migration: Cationic polymerization of 4,4-dimethyl-1-pentene. / G. Sartori, H. Lammens, Siffert J., A. Bernard // Journal of Polymer Science Part B: Polymer Letters. - 1971. - V 9. - I 8. - p. 599-603.

8. Синтез мономеров. // URL:https://vuzlit.ru/1132146/ sintez_monomerov.

9. Granovsky, Alex A. // Firefly version 8, http://classic. chem.msu.su/gran/firefly/index.html

10. General Atomic and Molecular Electronic Structure System / Schmidt M.W. [and others] // J. Comput. Chem. 1993. - №14. - P. 1347-1363.

11. Bode, B. M. MacMolPlt: A Graphical User Interface for GAMESS/. B.M. Bode and M.S. Gordon// J. Molec. Graphics. - 1998. - № 16. - P. 133-138.

12. Бабкин, В. А. Квантово-химический расчет некоторых молекул трифторметилстиролов методом DFT / В. А. Бабкин [и др.] // Фторные заметки. - 2019. - № 2 (123). - C. 5-6.

13. Бабкин, В. А. Квантово-химическое изучение механизма протонирования изоолефина 2-метилбутена-2 методом DFT / В. А. Бабкин [и др.] // Вестник Казан. технол. ун-та. - 2014. - Т. 17, № 2. - С. 12-17.

\section{REFERENCES}

1. Kennedy, J. Cationic polymerization of olefins / J. Kennedy. - M., 1978. - 431 p.

2. Edwards, W. R. Carbonium ion rearrangement in the cationic polymerization of branched alpha olefins / W. R. Edwards, Chamberlain N.F. // Journal of Polymer Science Part A: General Papers. -1963. - V1. - I 7. - p. $2299-2308$.

3. Goodrich, J.E. Cationic polymerization of 4-methyl-1pentene. / J.E. Goodrich, R. S. Porter // Journal of Polymer Science Part B: Polymer Letters. - 1964. - V 2. -№ 4. p. 353-357.

4. Wanless, G.G. Intramolecular hydride shift polymerization by cationic mechanism III - Structure analyses of deuterated and non-deuterated poly-4-methyl-1-pentene./ G.G. Wanless, J. P. Kennedy // Polymer. - 1965. - V. 6. p. $111-174$

5. Ketley, A.D. Isomerization polymerization of branched alpha olefins. / A.D. Ketley // Journal of Polymer Science Part B: Polymer Letters. - 1964. - V 2. - № 8. - p. 827-831.

6. Kennedy, J.P. Intramolecular hydride shift polymerization by cationic mechanism VII. The polymerization of 4-methyl-1-hexene / J.P Kennedy, J.J. Elliot, W. Naegele. // Journal of Polymer Science Part B: Polymer Letters. - 1965. - V 3. № 9. - p. $729-734$.

7. Sartori, G. A polymerization involving hydrogen and methyl group migration: Cationic polymerization of 4.4-dimethyl-1-pentene. / G. Sartori, H. Lammens, Siffert J., A. Bernard // Journal of Polymer Science Part B: Polymer Letters. -1971 . - V 9. - № 8. - p. $599-603$.

8. The synthesis of monomers. // URL: https: //vuzlit.ru/ 1132146/sintez monomerov. 
9. Granovsky, Alex A. // Firefly version 8, http: // classic. chem.msu.su/gran/firefly/index.html

10. General Atomic and Molecular Electronic Structure System / Schmidt M.W. [and others] // J. Comput. Chem. 1993. - No. 14. - p. 1347-1363.

11. Bode, B.M. MacMolPlt: A Graphical User Interface for GAMESS /. B.M. Bode and M.S. Gordon // J. Molec. Graphics. - 1998. - No. 16. - p. 133 - 138.
12. Babkin, V.A. Quantum chemical calculation of some trifluoromethylstyrene molecules by the DFT method. V.A. Babkin [and others] // Fluorine Notes. - 2019 . - №. 2 (123). - p. 5-6.

13. Babkin, V.A. Quantum-chemical study of the mechanism of protonation of the isoolefin 2-methylbutene-2 by DFT. / V.A. Babkin [and others] // Vestnik Kazan. technol. University. - 2014. - T.17, No. 2. - p. $12-17$.

V. A. Babkin ${ }^{1}$, D. S. Andreev ${ }^{1}$, A. V. Ignatov ${ }^{1}$, D. A. Borisov, R. O. Boldyrev ${ }^{1}$, T. V. Krapchetova ${ }^{1}$, M. V. Reshetnikova ${ }^{1}$, M. N. Kiseleva ${ }^{1}$, E. S. Titova ${ }^{2,3}$, A. I. Rakhimov ${ }^{2}$, V. S. Belousova ${ }^{4}$

\title{
CALCULATION OF THE ELECTRONIC STRUCTURE OF CATIONIC POLYMERIZATION MONOMERS BRANCHED IN THE $\beta$-POSITION TO THE DOUBLE BOND BY THE DFT METHOD
}

\author{
${ }^{1}$ Volgograd State Technical University, Sebraykov Branch \\ ${ }^{2}$ Volgograd State Technical University \\ ${ }^{3}$ Volgograd State Medical University of the Ministry of Health of Russia \\ ${ }^{4}$ I. M. Sechenov First Moscow State Medical University (Sechenov University)
}

Abstract. In this paper, a quantum chemical calculation of some monomers: 4-methylpentene-1, 4-methylhexene-1, 4,4-dimethylpentene-1 by the density functional DFT method is performed. The optimized geometric and electronic structure of these compounds is obtained. It was found that the studied monomers belong to the class of very weak $\mathrm{CH}$ acids $(\mathrm{pKa}=28)$.

Keywords: quantum chemical calculation, DFT method, 4-methylpentene-1, 4-methylhexene-1, 4,4-dimethylpentene-1, acid strength. 